\title{
Elaboração e validação de escala diagramática para a cercosporiose do pimentão
}

\author{
Sami Jorge Michereff ${ }^{1,2}$, Marissônia de Araújo Noronha ${ }^{1}$, Domingos Eduardo Guimarães Tavares de Andrade ${ }^{1}$, \\ Elaine Passos de Oliveira ${ }^{1}$, Maria Santina Xavier Filha ${ }^{1}$, Priscilla Anunciada Alves Moreira ${ }^{1}$
}

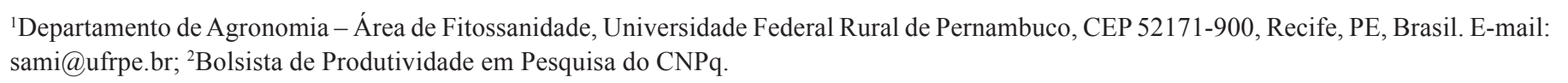
Autor para correspondência: Sami J. Michereff

Data de chegada: 25/05/2005. Aceito para publicação: 03/10/2005.

\section{ABSTRACT}

Michereff, S.J.; Noronha, M.A.; Andrade, D.E.G.T.; Oliveira, E.P.; Xavier Filha, M.S.; Moreira, P.A.A. Development and validation of a diagrammatic key for Cercospora leaf spot of sweet pepper. Summa Phytopathologica, v.32, n.3, p.260-266, 2006.

Cercospora leaf spot of sweet pepper caused by the fungus Cercospora capsici is an important disease occurring in tropical conditions. Due to the inexistence of standard methods to quantify this disease in field conditions, a diagrammatic key including the levels $1.5,3.5,8.0,16.5,31.0$ and $50.0 \%$ of diseased leaf area was elaborated and tested for accuracy, precision and reproducibility to estimate Cercospora leaf spot severity without and with using the key. The diagrammatic key was validated by 13 raters with and without using the key, using 50 leaves with different levels of severity previously measured by the software AutoCAD ${ }^{\circledR}$. Two evaluations were performed with the key at 7-day intervals when the same raters visually estimated different sequences of the same leaves. The accuracy and precision of each rater were determined by simple linear regression between actual and estimated severity. Without the key, eight raters overestimated disease severity. With the key raters obtained better levels of accuracy and precision, however four of them tended to overestimate the severity with absolute errors concentrated below $10 \%$. Raters had good repeatability and high reproducibility in estimating disease severity by using the key when it was not used. The proposed diagrammatic key is suitable for evaluation of sweet pepper Cercospora leaf spot severity.

Additional keywords: Capsicum annuun, Cercospora capsici, leaf disease, pathometry.

\section{RESUMO}

Michereff, S.J.; Noronha, M.A.; Andrade, D.E.G.T.; Oliveira, E.P.; Xavier Filha, M.S.; Moreira, P.A.A. Elaboração e validação de escala diagramática para a cercosporiose do pimentão. Summa Phytopathologica, v.32, n.3, p.260-266, 2006.

\begin{abstract}
A cercosporiose do pimentão, causada pelo fungo Cercospora capsici, é uma importante doença que ocorre em condições tropicais. Devido à inexistência de métodos padronizados para quantificação desta doença em campo, uma escala diagramática com os níveis de 1,5; 3,5; 8,$0 ; 16,5 ; 31,0$ e $50 \%$ de área foliar lesionada foi elaborada e testada para a acurácia, a precisão e a reprodutibilidade das estimativas de severidade da cercosporiose do pimentão, sem e com a sua utilização. Na validação da escala diagramática, 50 folhas com diferentes níveis de severidade da doença, mensurados previamente com o programa AutoCAD $^{\circledR}$, foram avaliadas por 13 avaliadores, sem e com a utilização da escala diagramática. Foram realizadas duas avaliações com utilização da escala, com intervalo de sete dias, onde seqüências
\end{abstract}

diferentes das mesmas folhas foram estimadas visualmente pelos mesmos avaliadores. A acurácia e a precisão de cada avaliador foi determinada por regressão linear simples, entre a severidade real e a estimada. Sem a escala, oito avaliadores superestimaram significativamente a severidade da doença. Com a escala, os avaliadores obtiveram melhores níveis de acurácia e precisão, embora quatro tendessem a superestimar a severidade, com os erros absolutos concentrando-se abaixo de $10 \%$. Os avaliadores apresentaram boa repetibilidade e elevada reprodutibilidade das estimativas com a utilização da escala, o mesmo não sendo verificado sem a utilização desta. A escala diagramática proposta mostrou-se adequada para avaliação da severidade da cercosporiose do pimentão.

Palavras-chave adicionais: Capsicum annuun, Cercospora capsici, doença foliar, patometria. 
A cercosporiose, causada pelo fungo Cercospora capsici Heald \& Wolf, é uma doença de ocorrência comum na cultura do pimentão (Capsicum annuun L.) em regiões quentes ou durante o verão, pois é favorecida por temperaturas acima de $25{ }^{\circ} \mathrm{C}$ e umidade do ar acima de $90 \%$. Plantas com estresse nutricional e hídrico são mais sensíveis à doença (12). Os sintomas da doença ocorrem principalmente nas folhas, que apresentam, inicialmente, lesões aquosas, translúcidas, de coloração verde-escura. Com o desenvolvimento da doença, as lesões tornam-se brancoacinzentadas, de formato circular, apresentando anéis concêntricos, com bordos escuros e centro cinza-claro. As lesões podem coalescer, formando grandes áreas necróticas, que se desprendem das folhas. Sob condições ambientais favoráveis, pode ocorrer intensa desfolha $(12,15)$, que favorece a exposição dos frutos diretamente à luz solar, bem como ao ataque de organismos secundários (19). Os sintomas podem também ser observados no caule, ramos e pedúnculos dos frutos, onde as lesões tendem a ser maiores e mais alongadas (12). As perdas ocasionadas pela cercosporiose podem ser grandes, principalmente após as primeiras colheitas. Os frutos das plantas atacadas são geralmente inadequados para comercialização, pois são pequenos, retorcidos e muitas vezes apresentam queimaduras por sol (15).

Considerando que o principal sintoma da cercosporiose do pimentão é foliar, sua intensidade é melhor expressa pela determinação da severidade, ou seja, porcentagem ou proporção de área foliar lesionada. Como a severidade das doenças é estimada visualmente, está sujeita a grande subjetividade e pode induzir a graves erros de acurácia, precisão e reprodutibilidade (22). A acurácia refere-se à proximidade de uma estimativa a um valor real de quantidade de doença avaliada, a precisão referese à confiabilidade e/ou repetibilidade associadas com uma estimativa e a reprodutibilidade refere-se à ausência de variação estatística significativa nas estimativas quando a mesma amostra de doença é avaliada por outro avaliador (2).

A avaliação da severidade da cercosporiose do pimentão tem sido realizada com o auxílio de escalas descritivas de notas (5, $19,24)$ e contagem do número de lesões por folha $(3,23)$. Os procedimentos de quantificação de doenças devem ser de fácil e rápida utilização para uma ampla gama de condições, mas também propiciar resultados acurados, precisos e reprodutíveis (2). Os métodos utilizados na quantificação da severidade da cercosporiose do pimentão apresentam várias limitações, motivo pelo qual o uso de uma escala diagramática poderá constituir uma excelente opção para minimizar a subjetividade das estimativas de severidade, além de auxiliar o avaliador.

Devido à inexistência de métodos padronizados para quantificação da cercosporiose do pimentão, este trabalho teve como objetivos desenvolver uma escala diagramática para quantificação da severidade da doença e analisar a qualidade das estimativas geradas com sua utilização.

\section{MATERIALEMÉTODOS}

\section{Elaboração da escala diagramática}

Para elaboração da escala diagramática foram coletadas 100 folhas de pimentão (cv. All Big) em plantios comerciais no município de Camocim de São Félix, PE, com diferentes níveis de severidade da cercosporiose. As folhas foram reproduzidas por fotocópias coloridas e digitalizadas. Com auxílio do programa
AutoCAD ${ }^{\circledR}$ (Autodesc, 1998), foram determinadas a área foliar total e a área necrótica de cada folha, obtendo-se a severidade da doença (porcentagem de área foliar lesionada). Utilizando o valor máximo de severidade da doença constatada nas folhas coletadas, os intervalos da escala foram calculados com o auxílio do programa 2LOG (22). Baseando-se na lei de Weber-Fechner de acuidade visual (9), bem como na forma, distribuição e freqüência das lesões, foi confeccionada uma escala diagramática logarítmica com seis níveis de severidade.

\section{Validação da escala diagramática}

No teste de validação da escala diagramática foram utilizadas 50 folhas de pimentão, reproduzidas em fotocópias coloridas, com sintomas de cercosporiose em diferentes níveis de severidade. A severidade foi estimada por 13 pessoas, a maioria sem experiência na quantificação de doenças, inicialmente, sem o auxílio da escala diagramática e, após sete dias, com o auxílio dessa. Visando avaliar a repetibilidade das estimativas com a escala diagramática, sete dias após a primeira avaliação, nova seqüência das mesmas folhas foi organizada e uma segunda estimativa visual foi efetuada com auxílio da escala pelos mesmos avaliadores.

A acurácia e a precisão de cada avaliador foi determinada por meio de regressão linear simples, com a severidade real obtida eletronicamente como variável independente e a severidade estimada pelo avaliador como variável dependente. A acurácia das estimativas de cada avaliador e do conjunto de avaliadores foi determinada pelo teste $t$ aplicado ao intercepto da regressão linear $(a)$, para verificar a hipótese Ho: $a=0$, e ao coeficiente angular da reta $(b)$, para testar a hipótese Ho: $b=1$, ao nível $5 \%$ de probabilidade $(\mathrm{P}=0,05)$. Valores de intercepto significativamente diferentes de 0 (zero) indicam superestimativa $(>0)$ ou subestimativa $(<0)$ da severidade real a níveis baixos de intensidade da doença, enquanto valores de coeficiente angular da reta que desviam significativamente de 1 (um) indicam superestimativa $(>1)$ ou subestimativa $(<1)$ sistemática da severidade real em todos os níveis de intensidade da doença (2, 17).

A precisão das estimativas foi determinada pelo coeficiente de determinação da regressão $\left(R^{2}\right)$, pela variância dos erros absolutos (severidade estimada menos severidade real) e pela repetibilidade das estimativas, determinada pela regressão da segunda avaliação em relação à primeira de uma mesma unidade amostral. A reprodutibilidade das estimativas foi determinada pelos valores de $R^{2}$ obtidos de regressões lineares entre as severidades estimadas da mesma unidade amostral por diferentes avaliadores combinados em pares $(2,10,17)$. As análises de regressão foram efetuadas com o auxílio do programa Microsoft Excel 2000 ${ }^{\circledR}$ (Microsoft Corporation, 2000).

\section{RESULTADOS E DISCUSSÃO}

O valor máximo de severidade da cercosporiose nas folhas de pimentão coletadas em plantios comerciais foi de $49,8 \%$. Valores acima de $50 \%$ são raramente encontrados no campo, pois causam senescência e queda prematura da folha.

A escala diagramática elaborada para quantificação da severidade da cercosporiose do pimentão foi representada pelos valores de 1,$5 ; 3,5 ; 8,0 ; 16,5 ; 31,0$ e 50,0\% de área foliar lesionada (Figura 1). Nos níveis de severidade a partir de $16,5 \%$, a 
representação dos sintomas incluiu tecidos necrosados e lesões coalescidas, como adotado na elaboração das escalas diagramáticas para cercosporiose da alface (7) e mancha de Alternaria em girassol (11). A coalescência de lesões é característica da cercosporiose do pimentão, que pode formar extensas áreas de tecido necrosado na folha (12).

$\mathrm{Na}$ validação da escala diagramática, oito avaliadores $(61,5 \%)$ foram pouco acurados quando a severidade da cercosporiose do pimentão foi estimada sem o auxílio da escala, apresentando valores do intercepto significativamente diferentes de zero para as retas de regressão entre a severidade real e a estimada, com valor médio de 4,36 (Tabela 1). Todos esses avaliadores superestimaram consistentemente a severidade, indicando a presença de desvios positivos constantes. O coeficiente angular da reta, na média dos avaliadores, foi de 1,36 e diferiu significativamente de 1 (Tabela 1). Para seis avaliadores (46,2\%) os valores do coeficiente angular foram significativamente diferentes de 1, indicando a presença de desvios sistemáticos em todos os níveis de intensidade da doença, com tendência à superestimativa (Tabela 1). A precisão das estimativas sem o auxílio da escala foi baixa, pois os coeficientes de determinação variaram entre 0,60 e 0,84 , com média de 0,70 . A distribuição dos resíduos das avaliações realizadas sem o auxílio da escala diagramática apresentou estimativas com erros absolutos variando entre-24,59 e 75,8 (Figura 2A).

Com a utilização da escala diagramática, a maioria dos

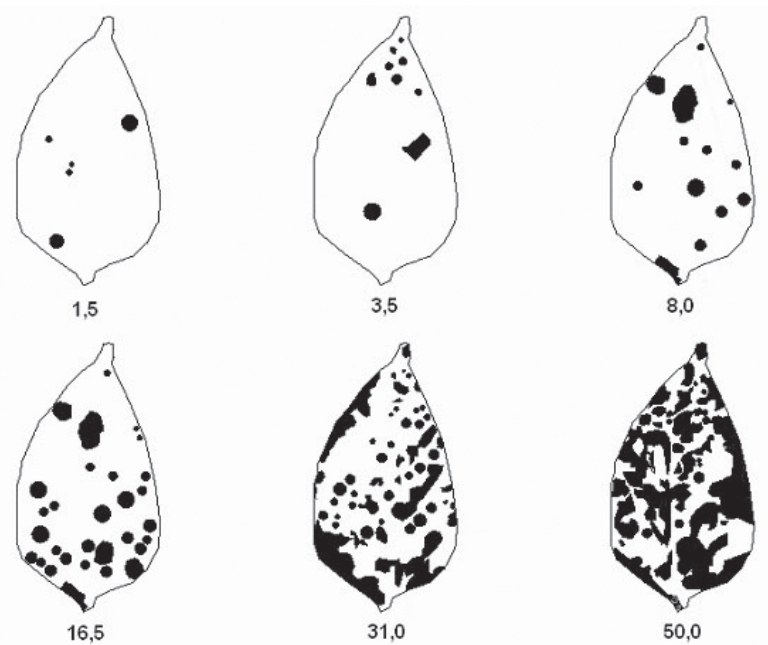

Figura 1. Escala diagramática para avaliação da cercosporiose do pimentão (Cercospora capsici), indicando os níveis de 1,5; 3,5; 8,0; 16,$5 ; 31,0$ e $50,0 \%$ de severidade.

avaliadores melhorou os níveis de acurácia e precisão das estimativas de severidade da cercosporiose do pimentão (Tabela 1). Na primeira avaliação com a escala, quatro avaliadores apresentaram valores do intercepto significativamente diferentes de zero, todos com desvios positivos constantes.

Tabela 1. Estimativa do intercepto $(a)$, do coeficiente angular da reta $(b)$ e do coeficiente de determinação $\left(R^{2}\right)$ de equações de regressão linear simples relacionando estimativas visuais da cercosporiose do pimentão (Cercospora capsici) efetuadas por treze avaliadores, sem e com o auxílio da escala diagramática, à severidade real determinada eletronicamente com auxílio do programa AutoCAD ${ }^{\circledR}$.

\begin{tabular}{|c|c|c|c|c|c|c|c|c|c|}
\hline \multirow{3}{*}{ Avaliador } & \multicolumn{4}{|c|}{ Sem Escala } & \multicolumn{5}{|c|}{ Com Escala } \\
\hline & \multirow[b]{2}{*}{$a$} & \multirow[b]{2}{*}{$b$} & \multirow[b]{2}{*}{$R^{2}$} & \multirow[b]{2}{*}{$a$} & \multicolumn{2}{|c|}{$1^{a}$ Avaliação } & \multirow[b]{2}{*}{$a$} & \multicolumn{2}{|c|}{$2^{a}$ Avaliação } \\
\hline & & & & & $b$ & $R^{2}$ & & $b$ & $R^{2}$ \\
\hline A & $2,14 *$ & $1,45^{*}$ & 0,66 & 0,31 & 1,09 & 0,89 & 0,07 & 1,08 & 0,90 \\
\hline $\mathrm{B}$ & $4,92 *$ & $2,26^{*}$ & 0,64 & 0,54 & 1,14 & 0,88 & 1,03 & 1,04 & 0,91 \\
\hline $\mathrm{C}$ & 1,00 & 1,14 & 0,74 & $1,86^{*}$ & 1,14 & 0,92 & 1,66 & 1,06 & 0,91 \\
\hline $\mathrm{D}$ & 1,66 & 1,13 & 0,74 & 0,92 & 1,07 & 0,89 & $-0,22$ & 1,03 & 0,90 \\
\hline $\mathrm{E}$ & $4,43^{*}$ & $1,17^{*}$ & 0,64 & 0,89 & 0,89 & 0,89 & 0,83 & 0,92 & 0,90 \\
\hline $\mathrm{F}$ & $-0,06$ & 0,98 & 0,74 & $-0,89$ & 1,06 & 0,91 & 0,93 & 0,96 & 0,91 \\
\hline G & $2,31 *$ & $2,22 *$ & 0,70 & $-0,12$ & 1,07 & 0,90 & 0,67 & 1,06 & 0,91 \\
\hline $\mathrm{H}$ & $2,54 *$ & 0,98 & 0,70 & $2,50 *$ & 0,92 & 0,91 & 0,31 & 1,02 & 0,90 \\
\hline I & $13,06^{*}$ & $1,27^{*}$ & 0,76 & 0,72 & 1,00 & 0,91 & 0,03 & 1,07 & 0,93 \\
\hline $\mathrm{J}$ & 0,95 & 1,12 & 0,84 & $2,22 *$ & 1,02 & 0,95 & 1,04 & 1,05 & 0,94 \\
\hline $\mathrm{K}$ & $18,10^{*}$ & $2,10^{*}$ & 0,69 & $2,37 *$ & 1,04 & 0,91 & $1,85^{*}$ & 1,10 & 0,92 \\
\hline $\mathrm{L}$ & $3,61^{*}$ & 1,00 & 0,67 & 0,16 & 1,08 & 0,92 & $-0,21$ & 1,02 & 0,92 \\
\hline $\mathrm{M}$ & 2,02 & 0,90 & 0,60 & 0,81 & 1,05 & 0,91 & 0,25 & 1,04 & 0,92 \\
\hline Média & $4,36^{*}$ & $1,36^{*}$ & 0,70 & 0,94 & 1,04 & 0,91 & 0,63 & 1,04 & 0,91 \\
\hline
\end{tabular}

*Asterisco representa situações onde a hipótese de nulidade $(a=0$ ou $b=1)$ foi rejeitada pelo teste $t(\mathrm{P}=0,05)$. 

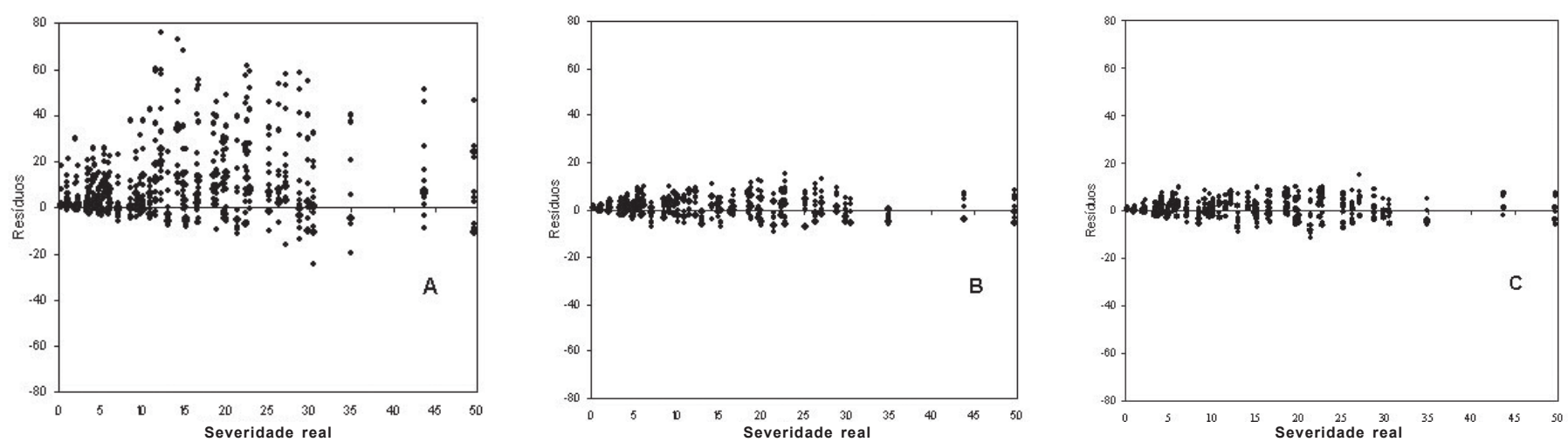

Figura 2. Distribuição dos resíduos (severidade estimada - severidade real) das estimativas da cercosporiose do pimentão (Cercospora capsici) realizadas sem o auxílio da escala diagramática (A) e com auxílio da escala diagramática na primeira (B) e segunda (C) avaliação.

Tabela 2. Estimativas do intercepto $(a)$, do coeficiente angular da reta $(b)$ e do coeficiente de determinação $\left(R^{2}\right)$ de equações de regressão linear simples relacionando a segunda à primeira estimativa da cercosporiose do pimentão (Cercospora capsici) por um mesmo avaliador, com o auxílio da escala diagramática.

\begin{tabular}{llll}
\hline Avaliador & $\boldsymbol{a}$ & $\boldsymbol{b}$ & $\boldsymbol{R}^{2}$ \\
\hline A & 0,00 & 0,97 & 0,97 \\
B & 1,46 & $0,86^{*}$ & $0,90,92$ \\
C & 0,54 & 0,89 & 0,86 \\
D & 0,01 & 0,89 & 0,96 \\
E & 0,22 & 1,02 & 0,88 \\
F & $2,55^{*}$ & $0,85^{*}$ & 0,90 \\
G & 1,59 & 0,93 & 0,85 \\
H & $-1,23$ & 1,02 & 0,91 \\
I & 0,14 & 1,00 & 0,90 \\
J & $-0,47$ & 0,99 & 0,90 \\
K & 0,31 & 1,01 & 0,89 \\
L & 0,43 & 0,89 & 0,89 \\
M & 0,33 & 0,93 & 0,90 \\
\hline Média & 0,45 & 0,94 & 0
\end{tabular}

*Asterisco indica que a hipótese de nulidade $(a=0$ ou $b=1)$ foi rejeitada pelo teste $t(\mathrm{P}=0,05)$.

Os valores médios do intercepto na primeira $(0,94)$ e na segunda $(0,63)$ avaliação não diferiram significativamente de zero (Tabela 1), indicando a redução dos erros verificados sem a utilização da escala.

A superestimativa dos níveis de severidade das doenças sem o uso de escalas diagramáticas, como verificado para a cercosporiose do pimentão, é comum em vários patossistemas $(10,16,21)$. A tendência dos avaliadores em superestimar os níveis de severidade da cercosporiose do pimentão quando utilizaram a escala diagramática assemelha-se ao constatado em vários estudos envolvendo validação de escalas diagramáticas $(4,8,11,18,20,21,22)$. No entanto, contrasta com o verificado na validação de escalas diagramáticas para cercosporiose da alface (7), queima das folhas do inhame (13), mancha parda da mandioca (14) e cancro bacteriano da videira (16), nos quais foi constatada a tendência dos avaliadores em subestimar os níveis de severidade das doenças com a utilização das escalas diagramáticas.

Em relação aos valores do coeficiente angular da reta, o uso da escala resultou em coeficientes angulares similares a 1 para todos os avaliadores nas duas avaliações, indicando a redução significativa dos erros sistemáticos e melhoria na acurácia das estimativas. Todos os avaliadores melhoraram a precisão das estimativas com a utilização da escala, com coeficientes de determinação variando entre 0,88 e 0,95 na primeira avaliação, e entre 0,90 e 0,94 na segunda, com média de 0,91 nas duas 
Tabela 3. Estimativas do coeficiente de determinação $\left(R^{2}\right)$ de equações de regressão linear simples relacionando as estimativas da cercosporiose do pimentão (Cercospora capsici) entre avaliadores sem o auxílio da escala diagramática e com auxílio da escala diagramática na primeira e segunda avaliação.

\begin{tabular}{|c|c|c|c|c|c|c|c|c|c|c|c|c|}
\hline \multicolumn{13}{|c|}{ Sem Escala } \\
\hline Avaliador & B & $\mathrm{C}$ & D & E & $\mathrm{F}$ & G & $\mathrm{H}$ & I & $\mathrm{J}$ & K & $\mathrm{L}$ & M \\
\hline A & 0,72 & 0,72 & 0,70 & 0,66 & 0,41 & 0,66 & 0,79 & 0,66 & 0,75 & 0,74 & 0,70 & 0,54 \\
\hline B & & 0,63 & 0,71 & 0,70 & 0,40 & 0,70 & 0,74 & 0,61 & 0,70 & 0,70 & 0,63 & 0,66 \\
\hline $\mathrm{C}$ & & & 0,67 & 0,66 & 0,42 & 0,64 & 0,76 & 0,64 & 0,79 & 0,60 & 0,59 & 0,66 \\
\hline $\mathrm{D}$ & & & & 0,79 & 0,51 & 0,69 & 0,73 & 0,73 & 0,83 & 0,74 & 0,69 & 0,65 \\
\hline $\mathrm{E}$ & & & & & 0,55 & 0,63 & 0,75 & 0,77 & 0,77 & 0,72 & 0,67 & 0,68 \\
\hline $\mathrm{F}$ & & & & & & 0,50 & 0,43 & 0,61 & 0,54 & 0,56 & 0,44 & 0,42 \\
\hline G & & & & & & & 0,76 & 0,55 & 0,70 & 0,66 & 0,68 & 0,57 \\
\hline $\mathrm{H}$ & & & & & & & & 0,75 & 0,79 & 0,74 & 0,78 & 0,75 \\
\hline I & & & & & & & & & 0,72 & 0,74 & 0,66 & 0,56 \\
\hline $\mathrm{J}$ & & & & & & & & & & 0,72 & 0,75 & 0,75 \\
\hline $\mathrm{K}$ & & & & & & & & & & & 077 & 0,61 \\
\hline $\mathrm{L}$ & & & & & & & & & & & & 0,61 \\
\hline
\end{tabular}

Com Escala - $1^{\text {a }}$. Avaliação

\begin{tabular}{|c|c|c|c|c|c|c|c|c|c|c|c|c|}
\hline Avaliador & B & $\mathrm{C}$ & $\mathrm{D}$ & $\mathrm{E}$ & $\mathrm{F}$ & G & $\mathrm{H}$ & I & $\mathrm{J}$ & $\mathrm{K}$ & $\mathrm{L}$ & M \\
\hline A & 0,86 & 0,93 & 0,85 & 0,86 & 0,84 & 0,85 & 0,82 & 0,85 & 0,87 & 0,90 & 0,86 & 0,90 \\
\hline B & & 0,93 & 0,91 & 0,79 & 0,81 & 0,91 & 0,93 & 0,89 & 0,90 & 0,88 & 0,88 & 0,87 \\
\hline $\mathrm{C}$ & & & 0,92 & 0,87 & 0,86 & 0,88 & 0,89 & 0,93 & 0,91 & 0,92 & 0,91 & 0,95 \\
\hline $\mathrm{D}$ & & & & 0,82 & 0,83 & 0,86 & 0,81 & 0,90 & 0,85 & 0,82 & 0,85 & 0,89 \\
\hline $\mathrm{E}$ & & & & & 0,90 & 0,81 & 0,82 & 0,84 & 0,87 & 0,84 & 0,84 & 0,82 \\
\hline $\mathrm{F}$ & & & & & & 0,83 & 0,83 & 0,84 & 0,90 & 0,81 & 0,85 & 0,83 \\
\hline G & & & & & & & 0,86 & 0,87 & 0,88 & 0,88 & 0,87 & 0,88 \\
\hline $\mathrm{H}$ & & & & & & & & 0,83 & 0,92 & 0,86 & 0,88 & 0,87 \\
\hline I & & & & & & & & & 0,88 & 0,87 & 0,90 & 0,90 \\
\hline $\mathrm{J}$ & & & & & & & & & & 0,88 & 0,90 & 0,89 \\
\hline $\mathrm{K}$ & & & & & & & & & & & 0,92 & 0,89 \\
\hline $\mathrm{L}$ & & & & & & & & & & & & 0,90 \\
\hline \multicolumn{13}{|c|}{ Com Escala - 2ª . Avaliação } \\
\hline Avaliador & B & $\mathrm{C}$ & $\mathrm{D}$ & E & $\mathrm{F}$ & G & $\mathrm{H}$ & I & $\mathrm{J}$ & $\mathrm{K}$ & $\mathrm{L}$ & M \\
\hline A & 0,80 & 0,85 & 0,87 & 0,85 & 0,84 & 0,87 & 0,85 & 0,90 & 0,87 & 0,83 & 0,88 & 0,87 \\
\hline B & & 0,92 & 0,89 & 0,86 & 0,84 & 0,90 & 0,91 & 0,88 & 0,93 & 0,90 & 0,87 & 0,90 \\
\hline $\mathrm{C}$ & & & 0,90 & 0,86 & 0,85 & 0,91 & 0,93 & 0,90 & 0,92 & 0,89 & 0,89 & 0,88 \\
\hline $\mathrm{D}$ & & & & 0,86 & 0,82 & 0,89 & 0,92 & 0,88 & 0,91 & 0,87 & 0,95 & 0,88 \\
\hline E & & & & & 0,89 & 0,85 & 0,84 & 0,85 & 0,85 & 0,83 & 0,87 & 0,89 \\
\hline $\mathrm{F}$ & & & & & & 0,85 & 0,83 & 0,86 & 0,87 & 0,85 & 0,89 & 0,85 \\
\hline $\mathrm{G}$ & & & & & & & 0,90 & 0,87 & 0,90 & 0,87 & 0,89 & 0,88 \\
\hline $\mathrm{H}$ & & & & & & & & 0,87 & 0,92 & 0,88 & 0,92 & 0,88 \\
\hline I & & & & & & & & & 0,94 & 0,89 & 0,90 & 0,87 \\
\hline $\mathrm{J}$ & & & & & & & & & & 0,90 & 0,92 & 0,88 \\
\hline $\mathrm{K}$ & & & & & & & & & & & 0,84 & 0,89 \\
\hline $\mathrm{L}$ & & & & & & & & & & & & 0,85 \\
\hline
\end{tabular}


avaliações (Tabela 1). Essa melhoria tem sido verificada com a utilização de outras escalas diagramáticas $(1,4,6,7,11,14,16$, $21,22)$, o que demonstra a importância dessa ferramenta em estudos de quantificação de doenças.

Houve uma redução nos erros absolutos para as estimativas com o auxílio da escala diagramática, quando comparada com a distribuição dos resíduos das estimativas obtidas sem a escala. Com a escala, os erros absolutos não apresentaram um padrão indesejável definido e variaram na primeira avaliação entre 9,40 e 12,90 (Figura 2B), com média de 1,52, enquanto na segunda avaliação entre-9,40 e 14,90 (Figura 2C), com média de 1,10. A maioria dos erros absolutos dos avaliadores que utilizaram a escala foi inferior a $10 \%$, considerado bom segundo os critérios adotados em vários estudos de validação de escalas diagramáticas. A presença de algum nível de erro absoluto nas mensurações pode ser compensada pela rapidez e padronização propiciadas pelo uso de escalas diagramáticas. Além disso, como a maioria dos métodos de quantificação de severidade de doenças, o uso de escalas diagramáticas está sujeito a um certo grau de subjetividade, o que pode ser minimizado com o treinamento dos avaliadores (17).

Em geral, os avaliadores evidenciaram boa repetibilidade nas estimativas da severidade da cercosporiose do pimentão com o uso da escala diagramática, pois a quantidade média de variação na primeira avaliação explicada pela segunda avaliação foi de $90 \%$ (Tabela 2). Os valores médios do intercepto e do coeficiente angular da reta não diferiram significativamente de zero e 1, respectivamente. Entre as duas avaliações, somente para um avaliador (F) o valor do intercepto foi significativamente diferente de zero e para dois avaliadores ( $\mathrm{B}$ e F) os valores do coeficiente angular da reta foram significativamente diferentes de 1 , indicando a elevada precisão das estimativas para a maioria dos avaliadores.

A reprodutibilidade das avaliações da severidade sem a utilização da escala diagramática foi baixa, pois as regressões lineares entre as severidades estimadas pelos 13 avaliadores produziram coeficientes de determinação variando de 0,40 e 0,83 , sendo que em somente $1,3 \%$ dos casos foi $\geq 0,80$ (Tabela 3). Por outro lado, com a utilização da escala diagramática as avaliações apresentaram elevada reprodutibilidade, pois nas duas avaliações os valores do coeficiente de determinação foram elevados, variando entre 0,79 a 0,95 , sendo $\geq 0,80 \mathrm{em} 100 \%$ dos casos nas duas avaliações (Tabela 3). O uso da escala diagramática aumentou a reprodutibilidade das estimativas obtidas por diferentes avaliadores e, do ponto de vista prático, diferentes experimentos, conduzidos por avaliadores distintos, serão comparáveis desde que utilizem a escala diagramática.

Os níveis de acurácia, precisão e reprodutibilidade das mensurações da severidade da cercosporiose do pimentão melhoraram significativamente com o uso da escala diagramática, indicando que sua adoção em estudos epidemiológicos poderá reduzir a subjetividade das estimativas e propiciar informações mais precisas sobre a intensidade da doença.

\section{AGRADECIMENTOS}

Os autores expressam seus agradecimentos a todos os avaliadores que participaram das etapas de validação da escala diagramática.

\section{REFERÊNCIASBIBLIOGRÁFICAS}

1. Amorim, L.; Bergamin Filho, A.; Palazzo, D.A.; Bassanezi, R.B.; Godoy, C.V.; Torres, G.M. Clorose variegada dos citros: uma escala diagramática para avaliação da severidade da doença. Fitopatologia Brasileira, Brasília, v.18, n.2, p.174-180, 1993.

2. Campbell, C.L.; Madden, L.V. Introduction to plant disease epidemiology. New York: John Wiley, 1990. 532p.

3. Cheema, D.S.; Singh, D.P.; Rawal, R.D.; Deshpande, A.A. Inheritance of resistance to Cercospora leaf spot in chilli. Vegetable Science, New Delhi, v.13, n.2, p.239-246, 1986.

4. Díaz, C.G.; Bassanezi, R.B.; Bergamim Filho, A. Desenvolvimento e validação de uma escala diagramática para Xanthomonas axonopodis pv. phaseoli em feijoeiro. Summa Phytopathologica, Jaboticabal, v.27, n.1, p.35-39, 2001.

5. Giordano, L.B.; Boiteux, L.S.; Lopes, C.A. Evaluation of fungicides for control of cercospora leaf spot on sweet pepper (Capsicum annuun). Fitopatologia Brasileira, Brasília, v.19, supl., p. 273, 1994. (Resumo).

6. Godoy, C.V.; Carneiro, S.M.T.P.G.; Iamauti, M.T.; Dalla Pria, M. Amorim, L.; Berger, R.D.; Bergamin Filho, A. Diagramatic scale for bean: development and validation. Zeitschrift für Pflanzenkrankheiten und Pflanzenschutz, Stuttgart, v.104, n.4, p.336-345, 1997.

7. Gomes, A.M.A.; Michereff, S.J.; Mariano, R.L.R. Elaboração e validação de escala diagramática para cercosporiose da alface. Summa Phytopathologica, Botucatu, v.30, n.1, p.39-43, 2004.

8. Hock, J.; Kranz, J.; Renfro, B.L. Tests of standard diagrams for field use in assessing the tarspot disease complex of maize (Zea mays). Tropical Pest Management, London, v.38, n.3, p.314318, 1992.

9. Horsfall, J.G.; Cowling, E.B. Pathometry: the measurement of plant disease. In: Horsfall, J.G.; Cowling, E.B. (Ed.). Plant disease: an advanced treatise - how disease develops in populations. New York: Academic Press, 1978. v.2, p.119-136.

10. Kranz, J. Measuring plant disease. In: Kranz, J.; Rotem, J. (Ed.). Experimental techniques in plant disease epidemiology. Heidelberg: Springer-Verlag, 1988. p.35-50.

11. Leite, R. M.V.B.C.; Amorim, L. Elaboração e validação de escala diagramática para mancha de Alternaria em girassol. Summa Phytopathologica, Botucatu, v. 28, n.1, p.14-19, 2002.

12. Matsuoka, K.; Vanetti, C.A.; Costa, H.; Pinto, C.M.F. Doenças causadas por fungos em pimentão e pimenta. Informe Agropecuário, Belo Horizonte, v.18, n.184, p.64-66, 1996.

13. Michereff, S.J.; Maffia, L.A.; Noronha, M.A. Escala diagramática para avaliação da severidade da queima das folhas do inhame. Fitopatologia Brasileira, Brasília, v. 25, n.4, 612-619, 2000.

14. Michereff, S.J.; Pedrosa, R.A.; Noronha, M.A.; Martins, R.B.; Silva, F.V. Escala diagramática e tamanho de amostras para avaliação da severidade da mancha parda da mandioca (Cercosporidium henningsii). Agrotrópica, Itabuna, n.3, p.143-148, 1998.

15. Monteiro, A.J.A.; Costa, H.; Zambolim, L. Doenças causadas por fungos e bactérias em pimentão e pimenta. In: Zambolim, L.; Vale, F.X.R.; Costa, H. (Ed.) Controle de doenças de plantas: hortaliças. Viçosa: Universidade Federal de Viçosa, 2000. v.2, p.637-675.

16. Nascimento, A.R.P.; Michereff, S.J.; Mariano, R.L.R.; Gomes, A.M.A. Elaboração e validação de escala diagramática para cancro bacteriano da videira. Summa Phytopathologica, Botucatu, v.31, n.1, p.59-64, 2005. 
17. Nutter Jr., F.W.; Schultz, P.M. Improving the accuracy and precision of disease assessments: selection of methods and use of computer-aided training programs. Canadian Journal of Plant Pathology, Ottawa, v.17, n.1, p.174-184, 1995.

18. Parker, S.R.; Shaw, M.W.; Royle, D.J. The reliability of visual estimates of disease severity on cereal leaves. Plant Pathology, London, v.43, n.6, p.856-865, 1995.

19. Sendín, M.A.P.; Barrios, J.G. Incidência de Cercospora capsici en zonas de producción de pimiento (Capsicum annuun) em Cuba. Ciencias de La Agricultura, Habana, v.21, p.19-24, 1984.

20. Shokes, F.M.; Berger, R.D.; Smith, D.H.; Rasp, J.M. Reliability of disease assessment procedures: a case study with late leafspot of peanut. Oléagineux, Paris, v.42, n.4, p.245-251, 1987.

21. Sposito, M.B.; Amorim, L.; Belasque Junior, J.; Bassanezi, R.B.; Aquino, R. Elaboração e validação de escala diagramática para avaliação da severidade da mancha preta em frutos cítricos.
Fitopatologia Brasileira, Fortaleza, v.29, n.1, p.81-85, 2004. 22. Tovar-Soto, A.; Hernandez-Martínez, M.; Cristóbal-Alejo, J.; Romero-Hijo, R.; Mora-Aguilera, G. Escala logarítimica diagramática de severidad de la mancha negra (Colletotrichum gloeosporioides Penz.) en Chirimoyo (Annona cherimola Mill). Revista Mexicana de Fitopatología, Sonora, v.20, n.1, p.103$109,2002$.

23. Ullasa, B.A.; Rawal, R.D.; Sohi, H.S.; Singh, D.P. Reaction of sweet pepper genotypes to anthracnose, cercospora leaf spot and powdery mildew. Plant Disease, St. Paul, v.65, n.7, p.600$601,1981$.

24. Vos, J. G. M.; Frinking, H.D. Nitrogen fertilization as a component of integrated crop management of hot pepper (Capsicum spp.) under tropical lowland conditions. International Journal of Pest Management, London, v.43, n.1, p.1-10, 1997. 